Classification

Physics Abstracts

$61.30 \mathrm{E}-64.70 \mathrm{E}$

\title{
Une nouvelle mésophase en colonnes
}

\author{
L. Mamlok, J. Malthete
}

Laboratoire de Chimie des Interactions Moléculaires, Collège de France, 75231 Paris Cedex, France

\author{
Nguyen Huu Tinh, C. Destrade
}

Centre de Recherche Paul-Pascal, Domaine Universitaire, 33405 Talence Cedex, France

et A. M. Levelut

Laboratoire de Physique des Solides (*), Bâtiment 510, 91405 Orsay, France

(Reçu le 24 mai 1982, accepté le 6 juillet 1982)

Résumé. - Nous décrivons un composé $(2 c)$ appartenant à une nouvelle famille de mésogènes

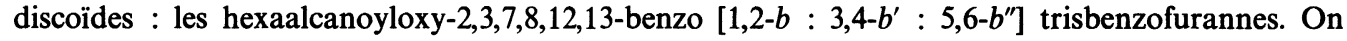
observe la séquence de phases suivante :

$$
\begin{array}{llllll}
K & N_{D} & D_{\text {ob.d }} & D_{\text {rd }} & D_{\text {hd }} & I
\end{array} \text {. }
$$

La phase $D_{\text {ob.d }}$ constitue le premier exemple d'une mésophase thermotrope en colonnes à réseau bidimensionnel oblique.

Abstract. - A compound (2c) of a new family of disc-like mesogens : 2,3,7,8,12,13-hexaalkanoyloxybenzo $\left[1,2-b: 3,4-b^{\prime}: 5,6-b^{\prime \prime}\right]$ trisbenzofurans is described with the phase sequence :

$$
\begin{array}{llllll}
K & N_{D} & D_{\text {ob.d }} & D_{\text {rd }} & D_{\text {hd }} & I
\end{array} \text {. }
$$

The $\mathrm{D}_{\text {ob.d }}$ phase is the first example of a thermotropic columnar mesophase with an oblique bidimensional lattice.

1. Introduction. - On a montré depuis peu que des composés discoïdes, dérivés hexasubstitués du benzène [1], du triphénylène [2-4] ou du truxène $[5,6]$ pouvaient présenter deux types de phases mésomorphes : une phase nématique $\left(\mathrm{N}_{\mathrm{D}}\right)$ [5-8] - et sa variante chirale cholestérique $\left(N_{D}^{*}\right)[9,10]$ et plusieurs phases en colonnes notées $D[11]$.

Ces dernières présentent un polymorphisme très diversifié $[4,12,13]$ : réseau bidimensionnel hexagonal de colonnes constituées d'un empilement ordonné $\left(D_{h o}\right)$ [14] ou désordonné des molécules $\left(\mathrm{D}_{\mathrm{hd}}\right)[15]$, réseau bidimensionnel rectangle avec empilement désordonné des molécules $\left(D_{r d}\right)[15]$; enfin, il existe une phase en colonnes où les molécules sont fortement inclinées $\left(\sim 55^{\circ}\right)$ par rapport à l'axe de la colonne [16].

(*) Associé au C.N.R.S. 
Jusqu'à présent, pour un même composé discoïde, on ne rencontre, au plus, que deux phases en colonnes différentes $[4-6,8,11-13,17,18]$.

Nous décrivons ici un représentant d'une nouvelle série de molécules discoïdes : l'hexa-ntétradécanoyloxy-2,3,7,8,12,13-benzo $\left[1,2-b: 3,4-b^{\prime}: 5,6-b^{\prime \prime}\right]$ trisbenzofuranne $\left({ }^{1}\right) 2 c$. Il présente un polymorphisme plus complexe : une phase nématique $\mathrm{N}_{\mathrm{D}}$ et trois phases en colonnes, dont une inconnue jusque-là.

\section{Préparation.}<smiles>COc1cc2c(cc1OC)C(=O)CO2</smiles>

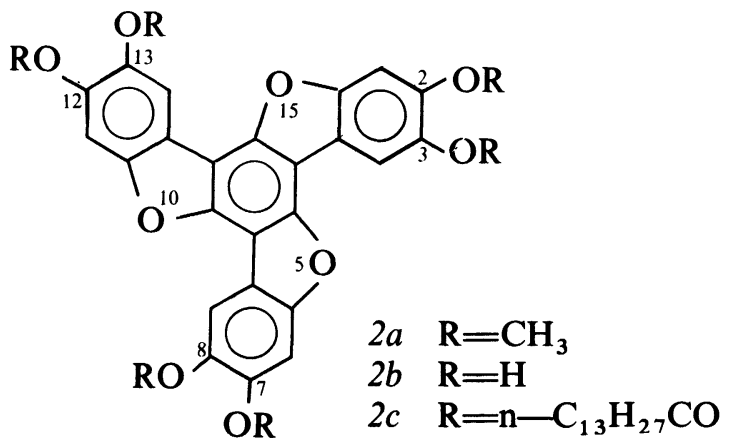

Le composé $2 a$ est obtenu par réaction dans le polyphosphate d'éthyle de la diméthoxy-5,6 $2 H$-benzofurannone-3 1 préparée elle-même par action du PPA sur l'acide diméthoxy-3,4 phénoxyacétique correspondant.

Par déméthylation de $2 a$ dans le chlorhydrate de pyridine, on accède à $2 b$ qui, condensé immédiatement après son obtention avec le chlorure d'acide approprié, par exemple le chlorure de n-tétradécanoyle, dans la pyridine anhydre, donne l'hexaester $2 c$ que l'on isole sur couche mince de silice (R.M.N. ${ }^{1} \mathrm{H}\left(\mathrm{CDCl}_{3}\right) \delta 0,85(t, 18 \mathrm{H}), 1,3(\mathrm{~m}, 120 \mathrm{H}), 1,84(\mathrm{~m}, 12 \mathrm{H}), 2,65(2 t, 12 \mathrm{H}), 7,61(\mathrm{~s}, 3 \mathrm{H})$, 8,08(s, 3H); I.R.(nujol) 1 765-1 615-1 150-1 $105 \mathrm{~cm}^{-1}$ ).

3. Résultats. - Le composé $2 c$ présente les phases et les transitions suivantes :

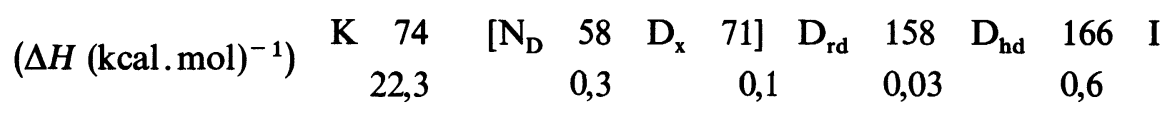

Les phases $N_{D}$ et $D_{r d}$ ont été identifiées par examen de leurs textures au microscope polarisant et par isomorphie avec des substances de référence. Les chaleurs de transition ont été déterminées au calorimètre (DUPONT 990). En l'absence d'une substance présentant une phase $\mathrm{D}_{\mathrm{hd}}$ dans un domaine de température compatible avec le dérivé décrit ici, la méthode de l'isomorphie n'a pu être utilisée pour confirmer la nature de la phase $D_{\text {hd }}$ du composé $2 c$. Et c'est seulement l'étude des diagrammes de diffraction des rayons $\mathrm{X}$ qui a permis d'élucider la structure de la nouvelle phase en colonnes $D_{\mathbf{x}}$.

4. Textures. - Par refroidissement du liquide isotrope, la phase $D_{\text {hd }}$ germine à partir d'un petit nombre de points de la préparation, puis se propage rapidement dans tout l'échantillon pour donner des figures en tous points semblables à celles obtenues dans le cas de la phase $D_{\text {hd }}$ des hexaalcanoyloxytriphénylènes à longues chaînes (Fig. 1a). En poursuivant le refroidisse-

$\left({ }^{1}\right)$ Le benzo [1,2-b : 3,4- $\left.b^{\prime}: 5,6-b^{\prime \prime}\right]$ trisbenzofuranne est l'analogue du truxène dont les trois méthylènes C-5, C-10 et C-15 sont remplacés par trois atomes d'oxygène. Par commodité, nous avons adopté la nomenclature des Chemical Abstracts. 


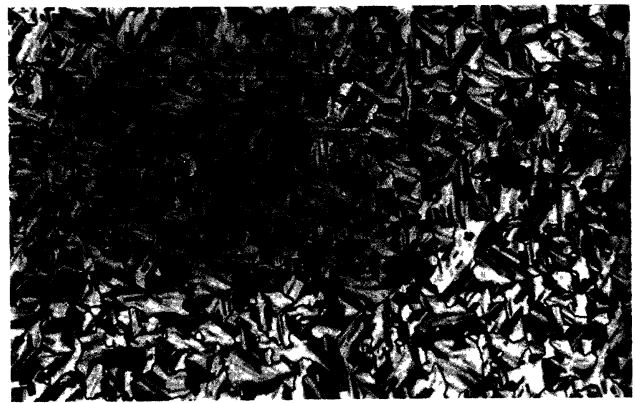

a)

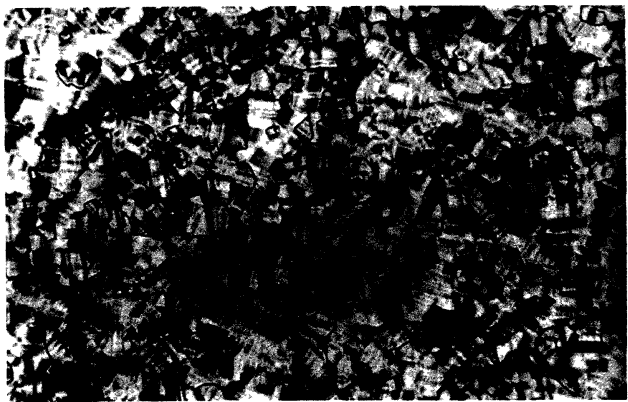




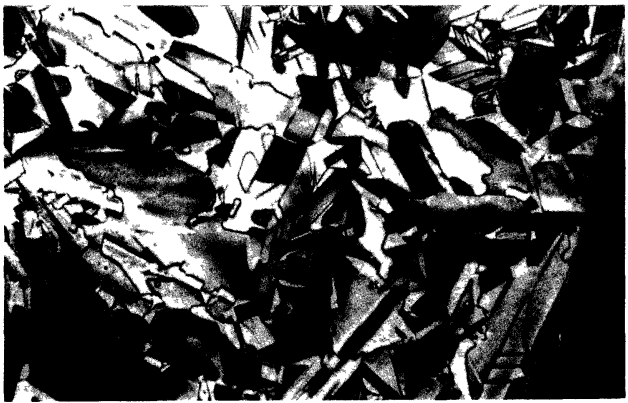

c)

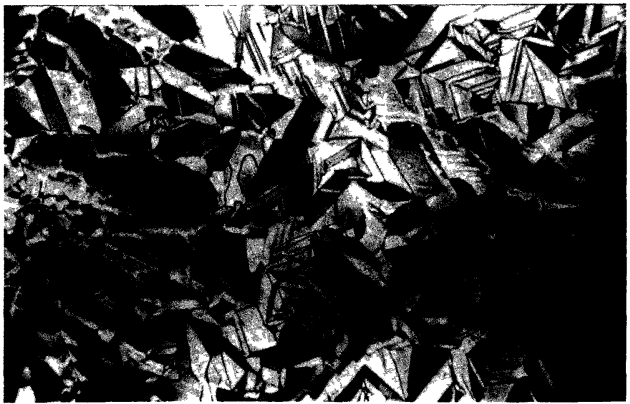




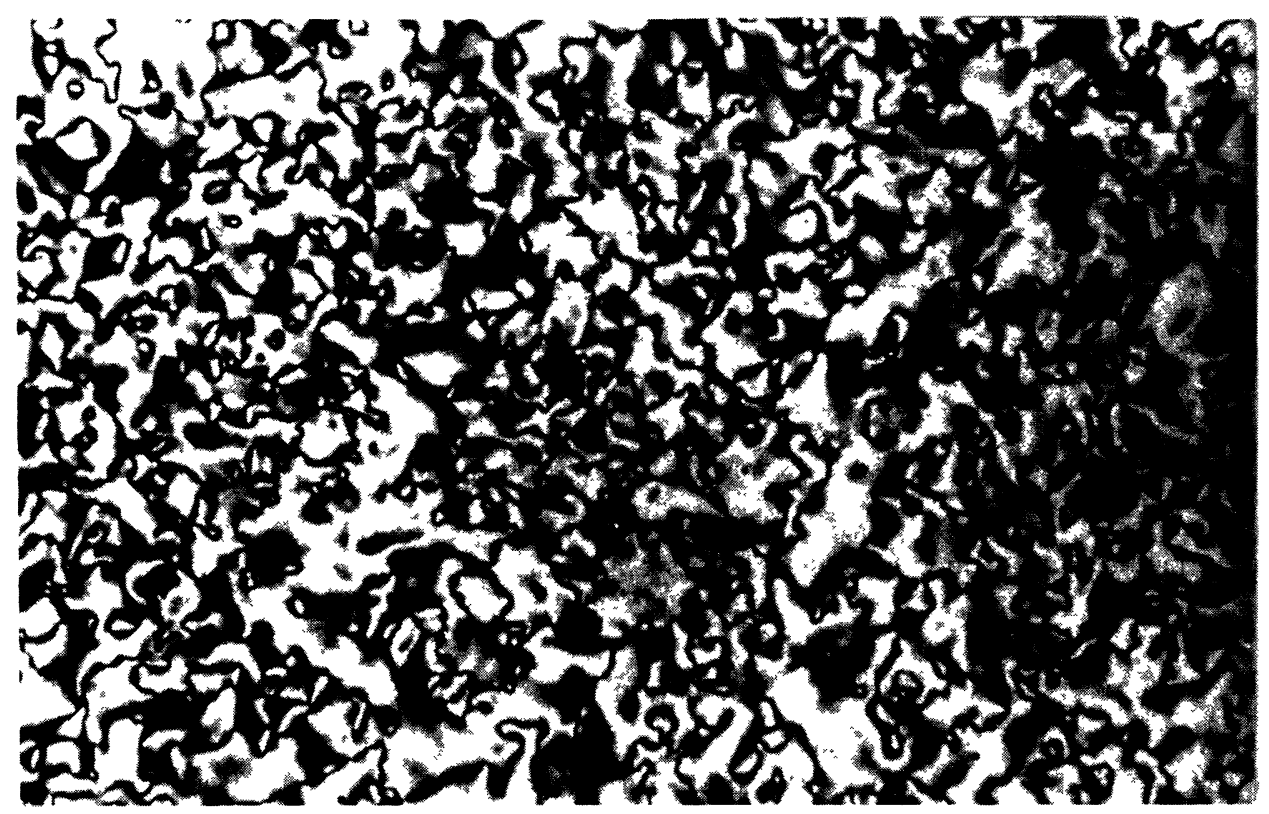

e)

Fig. 1. - Textures optiques du dérivé $2 c . a:$ Phase en colonnes $\mathrm{D}_{\text {hd }}$ à $163^{\circ} \mathrm{C}$. $b:$ Phase en colonnes $\mathrm{D}_{\text {rd }}$ à $144^{\circ} \mathrm{C} . c:$ Phase en colonnes $\mathrm{D}_{\mathrm{rd}}$ à $75^{\circ} \mathrm{C}$. $d:$ Phase en colonnes $\mathrm{D}_{\mathrm{ob} . \mathrm{d}}$ à $63^{\circ} \mathrm{C}$. $e:$ Phase nématique $\mathrm{N}_{\mathrm{D}}$ à $55^{\circ} \mathrm{C}$.

[Optical textures of the compound $2 c . a: \mathrm{D}_{\mathrm{hd}}$ columnar phase at $163^{\circ} \mathrm{C} . b: \mathrm{D}_{\mathrm{rd}}$ columnar phase at $144^{\circ} \mathrm{C}$. $c: \mathrm{D}_{\mathrm{rd}}$ columnar phase at $75^{\circ} \mathrm{C} . d: \mathrm{D}_{\mathrm{ob} . d}$ columnar phase at $63^{\circ} \mathrm{C} . e: \mathrm{N}_{\mathrm{D}}$ nematic phase at $55^{\circ} \mathrm{C}$.]

ment, le passage de la phase $D_{h d}$ à la phase $D_{r d}$ se traduit par l'apparition de stries concentriques caractéristiques de ce type de phases (Fig. 1b). Dans d'autres domaines, on peut observer des textures différentes (Fig. 1c). A température plus basse apparaît la phase $\mathrm{D}_{\mathrm{x}}$ avec une texture semblable à celle de la phase $\mathrm{D}_{\text {rd }}($ Fig. $1 c)$, mais avec une striation très nette des domaines développables et une biréfringence plus marquée (Fig. 1d). Enfin, à partir de $59{ }^{\circ} \mathrm{C}$ une phase très fluide à textures à fils est observée : c'est une phase nématique $\mathrm{N}_{\mathrm{D}}$ (Fig. 1e) qui, comme dans le cas des truxènes $[5,6]$, apparaît à plus basse température que les phases en colonnes. Il s'agit donc, ici, d'une nouvelle famille de mésogènes discoïdes où l'on peut observer une séquence inverse.

5. Diffraction des rayons $\mathbf{X}$. - Comme à l'accoutumée [15], nous avons réalisé deux types de diagramme :

- On observe, à l'aide d'un faisceau monochromatique ponctuel, les caractéristiques communes à toutes les phases en colonnes (précisons que les échantillons restent non orientés) : quelques anneaux fins apparaissent aux petits angles et caractérisent le réseau bidimensionnel des colonnes. En outre, un anneau large est observé pour une distance moyenne de 4,5 $\AA$ : il peut être relié à la structure "liquide " des chaînes paraffiniques. Enfin, il faut noter, dans les trois phases, l'absence d'un anneau fin qui traduirait un empilement régulier des molécules dans les colonnes [14].

- Pour déterminer avec certitude le réseau bidimensionnel formé par les colonnes, nous avons utilisé des diagrammes de poudre en chambre de Guinier : on retrouve respectivement les réseaux hexagonal et rectangle des phases $\mathrm{D}_{\mathrm{hd}}(a=30,0 \AA)$ et $\mathrm{D}_{\mathrm{rd}}(a=49,2 \AA$ et $b=29,3 \AA)$. 


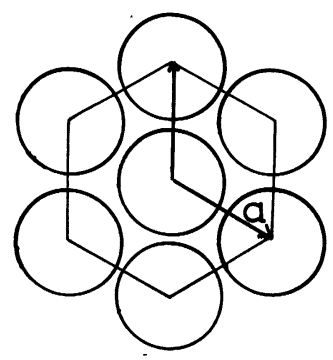

A

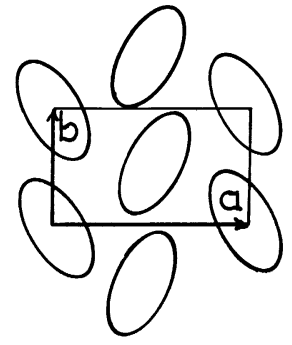

B

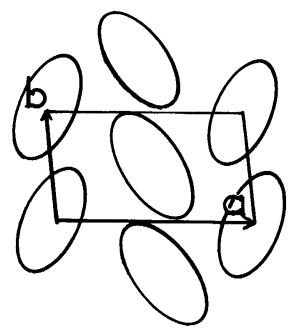

C

Fig. 2. - Représentation schématique des réseaux des phases en colonnes : A hexagonal; B rectangulaire ; $\mathrm{C}$ oblique.

[Schematic picture of the three columnar lattices : A hexagonal; B rectangular; C oblique.]

Le cliché obtenu dans la phase $\mathrm{D}_{\mathrm{x}}$ s'indexe. dans un réseau oblique $(a=49,7 \AA, b=28,0 \AA$, $\left.\gamma=97,5^{\circ}\right)$.

Le tableau I donne une liste des distances réticulaires observées pour les deux mésophases en colonnes apparaissant à basses températures. Remarquons que, dans la phase oblique, nous n'observons pas de raies ho d'ordre impair, ce qui semblerait indiquer la présence d'un miroir situé dans le plan du réseau avec glissement parallèle à $a$. Cependant, il est plus viraisemblable de supposer que la maille oblique qui contient deux colonnes s'obtient par déformation de la maille rectangle dont la surface est sensiblement identique : les colonnes que nous pouvons considérer de section elliptique seraient situées au sommet et au centre de la maille, les axes des ellipses faisant le même angle en valeur absolue avec la direction $b$ (Fig. 2).

Tableau I. - Distances réticulaires observées et calculées pour les deux mésophases en colonnes apparaissant à basse température.

[Observed and calculated reticular distances in the two low-temperature columnar mesophases.]

\begin{tabular}{|c|c|c|c|c|c|}
\hline \multicolumn{3}{|c|}{ Rectangle $T=76^{\circ} \mathrm{C}$} & \multicolumn{3}{c|}{ Oblique $T=68{ }^{\circ} \mathrm{C}$} \\
\hline \multirow{2}{*}{ Indices } & $\begin{array}{c}\text { Distances } \\
\text { mesurées }\end{array}$ & $\begin{array}{c}\text { Distances } \\
\text { calculées }\end{array}$ & Indices & $\begin{array}{c}\text { Distances } \\
\text { mesurées }\end{array}$ & $\begin{array}{c}\text { Distances } \\
\text { calculées }\end{array}$ \\
\hline 20 & 24,6 & 24,6 & 01 & 27,97 & 27,97 \\
11 & 25,15 & 25,15 & $1 \frac{1}{1}$ & 25,87 & 25,87 \\
21 & 18,65 & 18,13 & 20 & 24,86 & 24,86 \\
02 & 14,92 & 14,73 & $1 \frac{1}{1}$ & 23,83 & 23,11 \\
31 & 14,26 & 14,31 & 21 & 19,90 & 19,93 \\
40 & 12,44 & 12,57 & $2 \frac{1}{1}$ & 17,69 & 17,47 \\
22 & 12,04 & 12,30 & $\frac{32}{12}$ & 15,76 & 15,16 \\
& & & & - & 13,98 \\
& & & & & 13,95 \\
\hline
\end{tabular}

Remarques. - La raie 10 de la phase hexagonale est observée à $25,95 \AA$ pour $T=160^{\circ} \mathrm{C}$. Dans la phase oblique, on observe en outre quelques raies de faible intensité pour des dis- 
tances réticulaires inférieures à $14 \AA$; par exemple 41,03 et 42 . Mais beaucoup d'autres ne sont pas visibles dans le même domaine angulaire.

6. Conclusion. - Nous avons mis en évidence le premier exemple de réseau bidimensionnel oblique dans une phase en colonnes sur un nouveau motif mésogène discoïde. Compte tenu du désordre des molécules dans les colonnes et du réseau bidimensionnel oblique, nous proposons de noter cette nouvelle phase $D_{\text {ob.d }}$.

Remerciements. - Nous remercions M. P. Foucher qui a déterminé pour nous les enthalpies de transition.

\section{Bibliographie}

[1] Chandrasekhar, S., Sadashiva, B. K. et Suresh, K. A., Pramāna 9 (1977) 471.

[2] Nguyen Huu Tinh, Dubois, J. C., Malthete, J. et Destrade, C., C.R. Hebd. Séan. Acad. Sci. C286 (1978) 463.

[3] Billard, J. Dubois, J. C., Nguyen Huu Tinh et ŻanN, A., Nouv. J. Chim. 2 (1978) 535.

[4] Destrade, C., Mondon, M. C. et Malthete, J., J. Phys. C3 (1979) 17.

[5] Destrade, C., Malthete, J., Nguyen Huu Tinh et Gasparoux, H., Phys. Lett. 78A (1980) 82.

[6] Destrade, C., Malthete, J., Gasparoux, H., Babeau, A. et Nguyen HuU Tinh, Mol. Cryst. Liq. Cryst. 67 (1981) 37.

[7] Nguyen Huu Tinh, Destrade, C. et Gasparoux, H., Phys. Lett. 72A (1979) 251.

[8] Nguyen Huu Tinh, Gasparoux, H. et Destrade, C., Mol. Cryst. Liq. Cryst. 68 (1981) 101.

[9] Destrade, C., Nguyen Huu Tinh, Malthete, J. et Jacques, J., Phys. Lett. 79A (1980) 189.

[10] Malthete, J., Destrade, C., Nguyen HuU Tinh et Jacques, J., Mol. Cryst. Liq. Cryst. Lett. 64 (1981) 233.

[11] Destrade, C., Bernaud, M. C., Gasparoux, H., Levelut, A. M. et Nguyen HuU Tinh, Proceeding Int. Liq. Cryst. Conf. Bangalore (Heydon and Son, London) 1980, p. 29.

[12] Destrade, C., Mondon-Bernaud, M. C. et Nguyen Huu Tinh, Mol. Cryst. Liq. Cryst. Lett. 49 (1979) 169.

[13] Destrade, C., Nguyen Huu Tinh, Gasparoux, H., Malthete, J. et Levelut, A. M., Mol. Cryst. Liq. Cryst. 71 (1981) 111.

[14] Levelut, A. M., J. Physique-Lett. 40 (1979) L-81.

[15] Levelut, A. M., Proc. Int. Liq. Cryst. Conf. Bangalore (Heydon and Son, London) 1980, p. 21.

[16] Levelut, A. M., Hardouin, F., Gasparoux, H., Destrade, C. et Nguyen HuU Tinh, J. Physique 42 (1981) 147.

[17] Queguiner, A., Zann, A., Dubois, J. C. et Billard, J., Proc. Int. Liq. Cryst. Conf. Bangalore (Heydon and Son, London) 1980, p. 35.

[18] Billard, J., Dubois, J. C., Vaucher, C. et Levelut, A. M., Mol. Cryst. Liq. Cryst. 66 (1981) 115. 